\title{
Ajustes nomenclaturales en Geranium L. (Geraniaceae)
}

\author{
Carlos Aedo
}

Real Jardín Botánico, CSIC, Plaza de Murillo, 2, 28014 Madrid.

\author{
Correspondencia \\ C. Aedo \\ e-mail: aedo@rjb.csic.es \\ Recibido: 28 mayo 2017 \\ Aceptado: 4 diciembre 2017 \\ Publicado on-Iline: diciembre 2017
}

\author{
Nomenclatural ajustements in Geranium L. (Geraniaceae) \\ Palabras clave: Geranium, nomenclatura.
}

Kew words: Geranium, nomenclature.
La revisión de un considerable número de especies de Geranium L. en toda su área de distribución (Aedo 2008, 2012, 2017a, 2017b y referencias allí incluidas) ha permitido obtener una perspectiva global de la variabilidad género, a partir de la cual se puede proponer alguna modificación a la clasificación propuesta por Yeo (1984). Esta clasificación, que algunos autores han cuestionado por artificial, se ha mostrado muy sólida en sus aspectos centrales, de tal modo que hasta el momento no se ha propuesto alternativa alguna.

Yeo (1984) sugiere la división de Geranium en tres subgéneros atendiendo a la morfología del fruto. Dentro del subgénero Geranium, caracterizado por un fruto del tipo denominado "seed-ejection", en el que la semilla es lanzada lejos de la planta por un sistema de catapulta activado por la recurvatura de la arista y en el que el mericarpo queda unido a la arista, Yeo (1984) reconoce varios subtipos. Uno de ellos, denominado por dicho autor "Geranium bohemicum-type" es el que caracteriza al grupo que ahora proponemos como subgénero. En dicho tipo, la arista se desprende de la columela en el momento de la dispersión y la semilla se libera independientemente. Por otra parte el mericarpo de este grupo carece de callo en la base u otro mecanismo para prevenir la caída de la semilla en el estadío preexplosivo. Esto se logra por la torsión del mericarpo, que se dispone en un plano inclinado con respecto al eje principal del fruto. Otras características de este tipo de fruto son la amplia zona aplanada del ápice del mericarpo y la disposición helicoidal de las aristas en el rostro del fruto. Se trata de un conjunto muy importante de diferencias frente al tipo de fruto más general en Geranium L. subg. Geranium, que sugieren que estas plantas comparten un antepasado común con muy alta probabilidad y que por tanto constituyen un grupo natural. Este grupo de Geranium que se estaba clasificando hasta hora como sect. Tuberosa, se dividía de dos subgrupos, la subsect. Tuberosa que fue revisada taxonómicamente por Aedo \& Estrella (2006) y la subsect. Mediterranea que lo fue por Aedo et al. (2007). Como consecuencia, la sistemática de este grupo quedaría como sigue:

Geranium subg. Tuberosa (Boiss.) Aedo, subg. nov.

Geranium [unranked] Tuberosa Boiss., FI. Orient. 1: 869. 1867, basiónimo.- Holótipo: G. tuberosum L. (Art. 22.6 del ICN).

I. Geranium sect. Tuberosa (Boiss.) Reiche in Engl. \& Prantl, Nat. Pflanzenfam. 3(4): 8. 1890. Geranium [unranked] Tuberosa Boiss., Fl. Orient. 1: 869. 1867. Geranium subsect. Tuberosa (Boiss.) Yeo, Bot. J. Linn. Soc. 89: 12. 1984.-Holótipo: G. tuberosum L. (Art. 22.6 del ICN).

Especies incluidas: G. kotschyi Boiss., G. libanoticum A. Schenk, G. linearilobum DC., G. macrostylum Boiss., G. malviflorum Boiss. \& Reut., G. tuberaria Jacquem. ex Cambess. y $G$. tuberosum L.

II. Geranium sect. Lanuginosa Rouy in Rouy \& Fouc., Fl. France 4: 87. 1897.- Holótipo: G. lanuginosum Lam. (Art. 22.6 del ICN).

Geranium sect. Perennia Boiss., Diagn. PI. Orient. ser. 2, 1: 111. 1854, nom. nud.

Geranium subsect. Mediterranea R. Knuth in Engl., Pflanzenr. 53: 107. 1912.- Lectótipo: G. 
platypetalum Fisch. \& C.A. Mey. (designado por Yeo 1984: 12).

Geranium sect. Bohemica Tzvelev in Nov. Syst. PI. Vasc. 29: 95. 1993. - Holótipo: G. bohemicum L. (Art. 22.6 del ICN).

Especies incluidas: G. bohemicum L., $G$. gracile Ledeb., G. gymnocaulon DC., G. ibericum Cav., G. kurdicum Bornm., G. lanuginosum Lam., G. libani P.H. Davis, G. peloponnesiacum Boiss. y G. platypetalum Fisch. \& C.A. Mey.

Cuando Boissier (1854) describe $G$. peloponnesiacum indica que pertenece a la sect. Perennia, pero no proporciona ninguna descripción separada para dicha sección. Parecería lógica la extensión a otros rangos de las provisiones del art. 38.5 (Mcneil et al. 2012) para las descripciones genérico-específicas, pero el Código no lo contempla por el momento, de modo que el nombre seccional propuesto por Boissier ha de considerarse nomen nudum.

\section{Agradecimientos}

Este trabajo fue financiado por el proyecto Flora iberica (CGL2014-52787-C3-1-P). Se agradecen las sugerencias y comentarios de W. Greuter y A. Quintanar.

\section{Referencias}

Aedo, C. (2008). Geranium L. in Wu Zhengyi \& Raven, P.H. (eds.). Flora of China, 11, 7-30. Science Press.

Aedo, C. (2012). Revision of Geranium L. (Geraniaceae) of the New World. Syst. Bot. Monogr. 95 (550 págs.).

Aedo, C. (2017a). Revision of Geranium L. (Geraniaceae) in the Pacific area. Syst. Bot. Monogr., 102 (240 págs.).

Aedo, C. (2017b). Taxonomic revision of Geranium Sect. Ruberta and Unguiculata (Geraniaceae). Ann. Missouri Bot. Gard., 102, 409-465.

Aedo, C. \& M. Estrella, (2006). Taxonomic revision of Geranium subsect. Tuberosa (Boiss.) Yeo (Geraniaceae). Israel J. Pl. Sci., 54(1), 19-54.

Aedo, C., García, M.A. , Alarcón, M.L., Aldasoro, J.J. \& Navarro, C. (2007). Taxonomic revision of Geranium subsect. Mediterranea (Geraniaceae). Syst. Bot., 32, 93-128.

Boissier, É. (1854). Diagnoses Plantarum Novarum ser. 2, 3(1), 1-120. Neocomi, typis H. Wolfrath.

Mcneill, J., Barrie, F.R., Buck, W.R., Demoulin, V., Greuter, W., Hawksworth, D.L., Herendeen, P.S., Knapp, S., Marhold, K., Prado, J., Prud'homme Van Reine, W.F., Smith, G.F., Wiersema, J.H. \& Turland, N. J. (2012). International Code of Nomenclatureforalgae, fungi, and plants (Melbourne Code). Regnum Veg., 154 pp.

Yeo, P.F. (1984). Fruit-discharge-type in Geranium (Geraniaceae): its use in classification and its evolutionary implications. Bot. J. Linn. Soc., 89, 1-36. 